\title{
DISEÑO SOSTENIBLE EN EL DESARROLLO DE LA U.S.H.S.R.S. EN NUEVA ESPAÑA
}

\author{
Luis Fort López-Tello \\ Dr. Ingeniero de Caminos, Canales y Puertos, Dr Ingeniero Agrónomo. Jubilado de los \\ Cuerpos de Ingenieros de Caminos del Estado y de Profesores Titulares de Universidad. \\ Madrid. e-mail: Ifort@ ciccp.es

\section{Carmen Fort Santa-María} \\ Dr. Ingeniero de Caminos, Canales y Puertos, Master en Gestión de Infraestructuras, \\ Equipamientos y Servicios. Jefe de Proyectos de la División de Infraestructura del \\ Transporte, EPTISA, S.I. Madrid, e-mail: cfort@eptisa.com
}

\section{RESUMEN}

Se propone como legislación básica de la política de transportes para aplicar a la red de alta velocidad ferroviaria de Estados Unidos (USHSRS), una ley federal como la de Suiza (enclave de las infraestructuras transalpinas) LPE (Loi sur la protection de l'environnement).

"Esta ley tiene por objeto proteger a los hombres, los animales y las plantas, sus biocenosis y sus biotopos", de forma que resulte incuestionable que la legislación debe privilegiar la protección de los seres vivos que viven en interacción con sus medios. El conjunto de estos elementos y de sus actividades forma un ecosistema cuya supervivencia y equilibrio están asegurados si lo están la salud y la calidad de los componentes y de sus intercambios. Este sistema desarrolla su actividad en un espacio dado, caracterizado por su geomorfología y su clima. El estudio de las modificaciones que para el desarrollo de esta actividad haya que introducir en ese espacio debe empezar por dar orden de preferencia a los impactos generados. El primer grupo de éstos reúne aquellos elementos que puedan poner directamente en peligro la supervivencia y la salud e integridad del hombre.

Una aplicación de este principio al diseño de infraestructuras lineales resulta particularmente interesante en el mayor reto mundial de ingeniería civil de los próximos años, que supone el desarrollo del Plan de Alta Velocidad Ferroviaria de Estados Unidos (USHSRS) desde su costa del Pacífico hasta llegar a las grandes llanuras del Mississippi, tras atravesar el sistema de las Rocosas, en el inmenso Oeste norteamericano que constituyó en los siglos XVI a XIX la antigua Nueva España.

\section{BASES DE DISEÑO}

Consecuentemente con el principio antes expuesto, el diseño de las infraestructuras de las líneas ferroviarias de alta velocidad (HSRL) debe basarse en el respeto ordenado del ecosistema al que afectan, intentando garantizar al máximo los siguientes requerimientos: $1^{\circ}$-Seguridad y Confort ("presente" del hombre) $2^{\circ}$ - Respeto al patrimonio histórico ("pasado" del hombre) $3^{\circ}$ Sostenibilidad ("futuro " del hombre) "The land belongs as much to the Living, as to the Dead and the Unborn". (Figuras 1 y 2 ) 

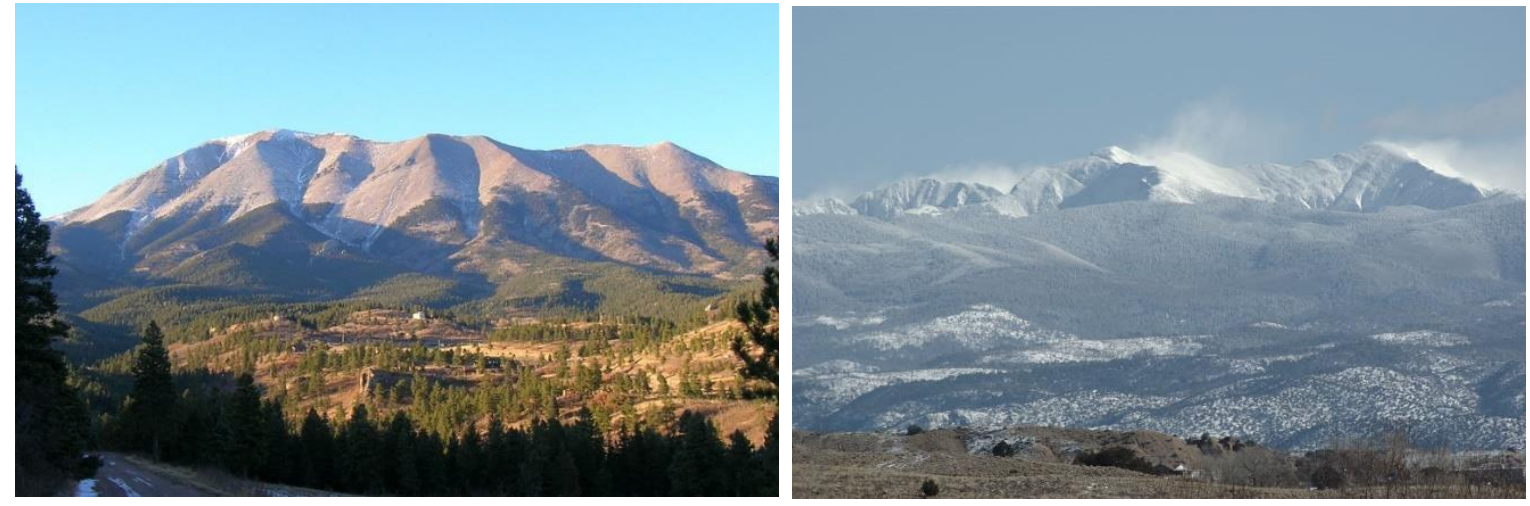

Figura 1. Truchas Peak y Pico Español en Sangre de Cristo Mountains (Santa Fe National Forest) New Mexico
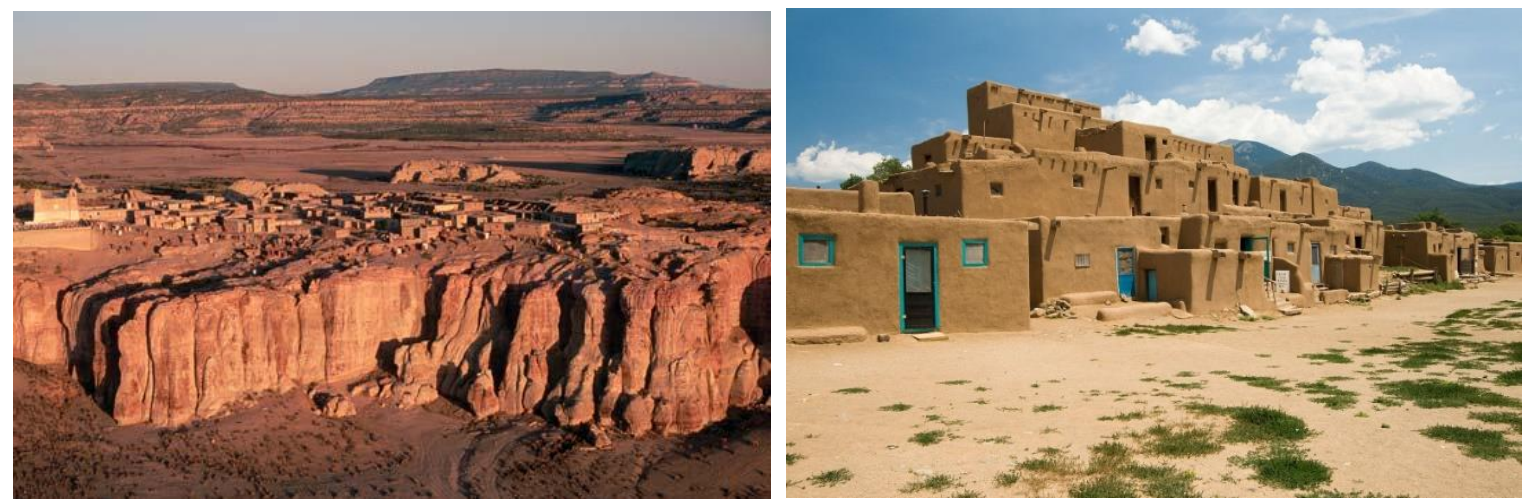

Figura 2. Ácoma (camino del gobernador Juan de Oñate "El Adelantado") Indian Reservation

El requerimiento de seguridad se considera prioritario, pues protege al usuario del transporte, el hombre, núcleo y razón de ser del entorno a proteger y por otra parte elemento condicionante para el tercer requerimiento de sostenibilidad, de forma que no constituya su falta de garantía un elemento disuasorio de utilización de esta infraestructura viaria, que a su vez dificulte la rentabilidad de su explotación.

\section{ELEMENTOS DE DISEÑO}

En aquellos tramos de líneas comprendidos entre dos PAETs que discurren por terrenos de relieve "accidentado/muy accidentado" y que por tanto su trazado requiere el paso con túneles largos y viaductos de luz importante (Figura 3), se estima decisivo para dotar a la explotación de esos tramos de una máxima seguridad fiable, reducir al máximo posible (incluso aumentando las pendientes, siempre que sean compatibles con las reducciones de velocidad establecidas con los condicionantes de explotación) la longitud de los túneles, disponer éstos de una gran sección libre, permitir la explotación en doble vía en todo el tramo, sin limitaciones ni condicionantes y finalmente disponer en todo el recorrido de los túneles de otro túnel auxiliar, paralelo y comunicado con el principal (Figura 4), para circulación de trenes de evacuación (VAL), que de forma sistemática sigan el paso de los trenes de explotación (TAV) de línea que circulan por los túneles principales, así como la del acceso rodado de vehículos de salvamento, rescate y extinción de incendios. Partiendo en cada caso de un detenido estudio de estrategias de evacuación en el tramo entre PAETs correspondiente, se determina el tiempo de evacuación con las condiciones pésimas de rescate. Así por ejemplo, en el Proyecto Farwest de la CHSRS (California High Speed Railway System) en la HSRL "Golden Gate" Fresno- San Francisco en el tramo PAET 
Oroloma- HSR Gilroy Station $(75,85 \mathrm{~km})$, que cruza la "Diablo Range" con tres túneles: (TU1 de 16,2 km, en rampa sentido Oroloma-Gilroy de 7,16 milésimas), (TU2 de 19,2 km, doble pendiente, rampa de 2,75 milésimas en $4 \mathrm{~km}$ desde su boca Este y pendiente de 10,45 milésimas en 15,2 km hasta su boca Oeste) y (TU3 de 9,2 km, todo en pendiente de 6,5 milésimas hacia Gilroy), los tiempos de evacuación en condiciones pésimas de rescate (a $750 \mathrm{~m}$ de las bocas de salida) son de $11 \mathrm{~min} 20$ s (accidente en el TU1), $26 \mathrm{~min} 36 \mathrm{~s}$ (accidente en el TU2) y $29 \min 36 \mathrm{~s}$ (accidente en el TU3).

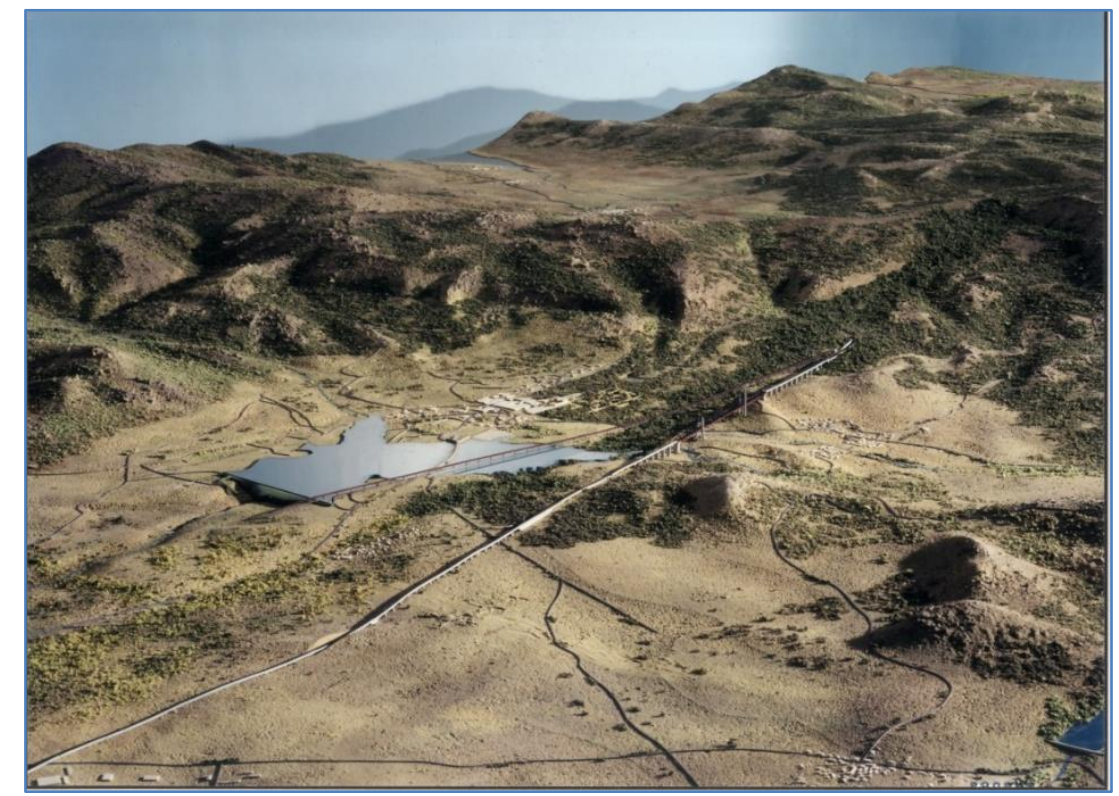

Figura 3. Sierra de Guadarrama LAV Madrid-Segovia. Alternativa Paraíso (Maqueta ETSICCP Madrid)

Para permitir la circulación de los trenes TAV en todo el tramo es necesario, para continuidad de la plataforma de circulación de los trenes de auxilio (VAL), dotar a la sección de los viaductos (Figura 5), de un segundo nivel en el tablero, bajo las vías principales, para alojar la vía de los trenes de auxilio. En los tramos intertúneles, a cielo abierto, la plataforma se amplía, y en el recorrido de los túneles, los trenes VAL circulan por un segundo tubo auxiliar de sección más reducida (6,35 m de diámetro interior, Figura 4).

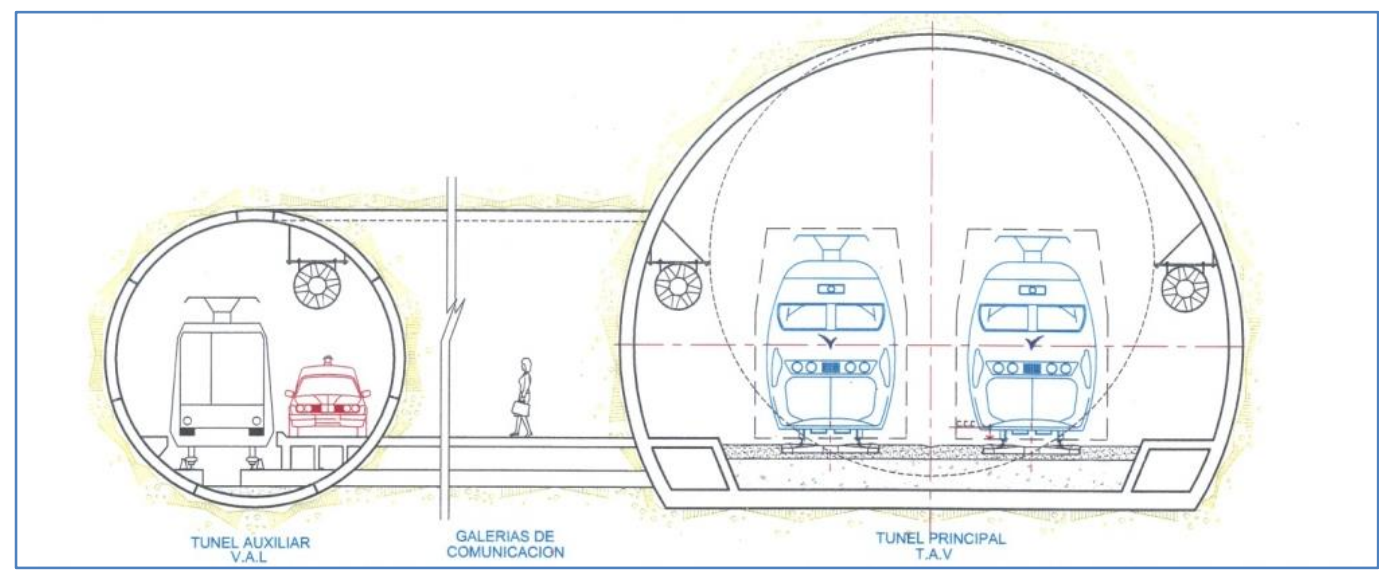

Figura 4. Túnel Principal (TAV) doble vía y Túnel auxiliar (VAL) 

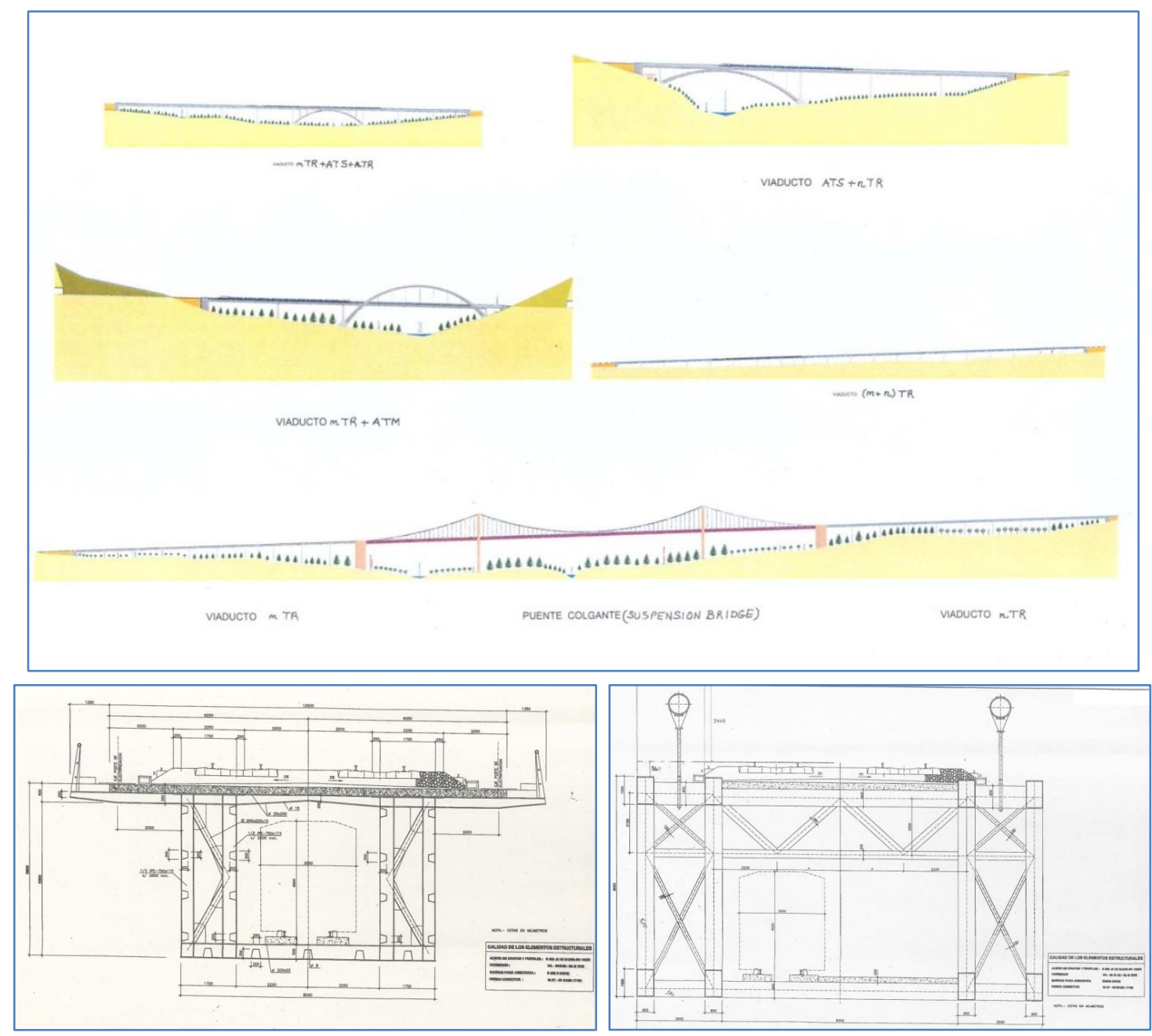

Figura 5. Tipología de Grandes Viaductos y Sección con nivel VAL

El dimensionamiento de las secciones de los túneles principales, derivado de las condiciones aerodinámicas de seguridad y confort para circular a $350 \mathrm{~km} / \mathrm{h}$ requiere adoptar para los túneles largos de doble vía una sección libre de $101 \mathrm{~m}^{2}$ (sección de excavación de $134 \mathrm{~m}^{2}$ ) en los 750m extremos y una sección libre de $86 \mathrm{~m}^{2}$ (sección de excavación de 100 m²) en el resto del tramo central.

En los túneles de vía única, para el cumplimiento estricto de la condición de confort establecida en las Bases Técnicas de Concursos de la Administración Ferroviaria española, variación máxima de $2,5 \mathrm{kPa}$ en $4 \mathrm{~s}$, con sección transversal de tren de $8 \mathrm{~m}^{2}$ y velocidad de $300 \mathrm{~km} / \mathrm{h}$, resulta una sección libre de túnel de $58,2 \mathrm{~m}^{2}$, que requiere un diámetro interior mínimo en sección circular de 9m (calculada con el programa Thermotun) $\mathrm{S}$ excavación $=$ $63,62 \mathrm{~m}^{2} \mathrm{o}$ de $10 \mathrm{~m}$ (con el programa Ouranos) Sexcavación $=78,54$ m².

\section{CONSIDERACIONES ECONÓMICAS DE DISEÑO}

El Sistema de las Rocosas ocupa más de un tercio del territorio estadounidense (Figura 6). Formadas en el transcurso de largos períodos geológicos, el levantamiento de su parte 
oriental que es la más antigua se efectuó en el Jurásico. Rocas antiguas, cristalinas y sedimentarias afloran por todas partes. Las Rocosas centrales que comprenden la Wasatch Range y los montes Uinta están constituidas por rocas cenozoicas y al igual que en la meseta de Colorado están depositadas sobre el zócalo de rocas cristalinas (gneis y esquistos antiguos). El río Colorado las tajó, formando el Gran Cañón que en su corte de hasta 1800m de profundidad permite distinguir todos los estratos de estas formaciones: partiendo de abajo, por encima de los granitos rosados del Pérmico y del Triásico antiguo, después las blancas verduscas o amarillas del Triásico reciente y del Jurásico y más arriba las importantes formaciones color amarillo y negruzco del Cretácico y por último los tintes rosados y naranja de las rocas del Cenozoico.

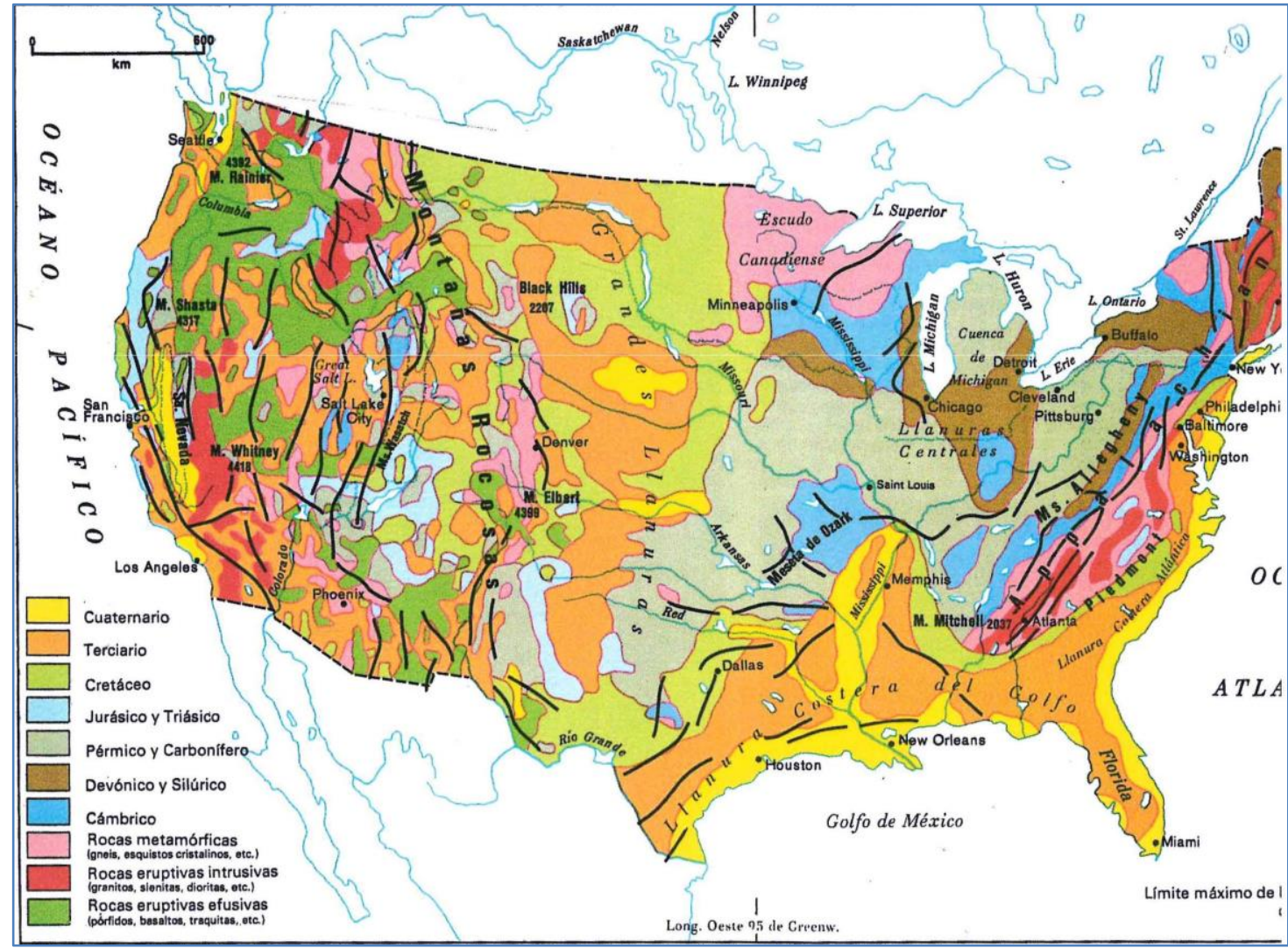

Figura 6. Geología de los Estados Unidos. Sistema de las Rocosas

Tomando como base información de costes unitarios homogeneizados (excavados con TBM en rocas variadas, pero de perforabilidad media similar a las de cotas altas de Nueva España) de túneles ferroviarios de líneas de alta velocidad española, se pueden establecer las siguientes valoraciones medias para túneles de longitud "L", con la ayuda de algunos parámetros y consideraciones que se comentan a continuación:

-Para tener en cuenta el peso diferente, según la longitud del túnel, que aun suponiendo aplicable un mismo coste medio unitario, tienen las distintas secciones de las zonas extremas y la central, se define también en cada túnel de longitud total L, una longitud equivalente "Leq":

Leq $=(2 * 0,75 * 1,34+(\mathrm{L}-1,5)) / 1,24=0,8065 \mathrm{~L}+0,4113$

Túneles vía única: $=28,878 * 1+14,792 \mathrm{M} \$$

Túneles vía doble: $=24,195^{*} \mathrm{~L}+12,393 \mathrm{M} \$$

Auxiliar: 7,60*L M\$ Galerías de evacuación: 0,46*L M\$ 
Suplementos plataforma VAL:

Túneles:

Principales (Trenes TAV): $\quad 4,683 * \mathrm{~L}+2,399 \mathrm{M} \$$

Auxiliar (Trenes VAL):.................7,60*L M\$

Galerías de evacuación.................0,46* L M\$

Grades Viaductos:

Tramo recto $(\mathrm{L}=100 \mathrm{~m})$

Arco Tablero Superior $(\mathrm{L}=200 \mathrm{~m}) \ldots . .2,990 \mathrm{M} \$$

Arco Tablero Intermedio (L=200m) 3,133 M\$

Arco Tablero Inferior...(L=200m) 3,133 M\$

Suspension Bridge $(\mathrm{L}=800 \mathrm{~m}) \quad 27,483 \mathrm{M} \$$

Infraestructura a cielo abierto:

20\% Movimiento de Tierras Plataforma doble vía

Superestructura:

$0,394 \mathrm{M} \$ / \mathrm{km}$

Instalaciones:

$0,428 \mathrm{M} \$$

Resto Capítulos:

$8,77 \%$ (Capítulos anteriores)

\section{ESTIMACIÓN DE SOBRECOSTO DE DISEÑO EN LÍNEAS CON GRANDES TÚNELES}

En el Proyecto FARWEST, Alternativa de la CHSRS propuesta por los autores de esta Ponencia, se ha aplicado la medida descrita para el aumento de seguridad en trazado de líneas de alta velocidad al atravesar sistemas montañosos, proyectando solución monotubo de gran sección para alojar plataforma de doble vía para circulación de trenes TAV en los dos sentidos, en lugar de solución bitubo de sección estricta para la circulación de los trenes TAV en cada sentido. La evacuación en caso de accidente en el interior de un túnel se hace igualmente con galerías de escape espaciadas a no más de $500 \mathrm{~m}$. En el caso bitubo, comunicando ambos túneles de circulación de trenes TAV, lo que implica que no pueda hacerse el rescate de los pasajeros del tren accidentado, manteniendo el servicio de la línea en los dos sentidos de circulación sin interrupción y retrasando por tanto unos minutos críticos su rescate y evacuación. En el caso de túnel único de gran sección, las galerías de escape comunican con otro túnel auxiliar de menor sección por el que circulan los trenes VAL que siguen a velocidad de $100 \mathrm{~km} / \mathrm{h}$ a cada tren TAV que pasa por agujas en uno de los PAETs, que limitan el trayecto con plataforma para la circulación de los trenes VAL, "Plataforma VAL".

En el texto y figuras adjuntas se reproducen (tomadas de tres líneas del Proyecto Farwest) las estrategias de evacuación en tres casos de accidente, con tiempos de rescate, inferiores a la media hora, que se estima suficiente para que sea efectivo en condiciones contrastadas de supervivencia. 


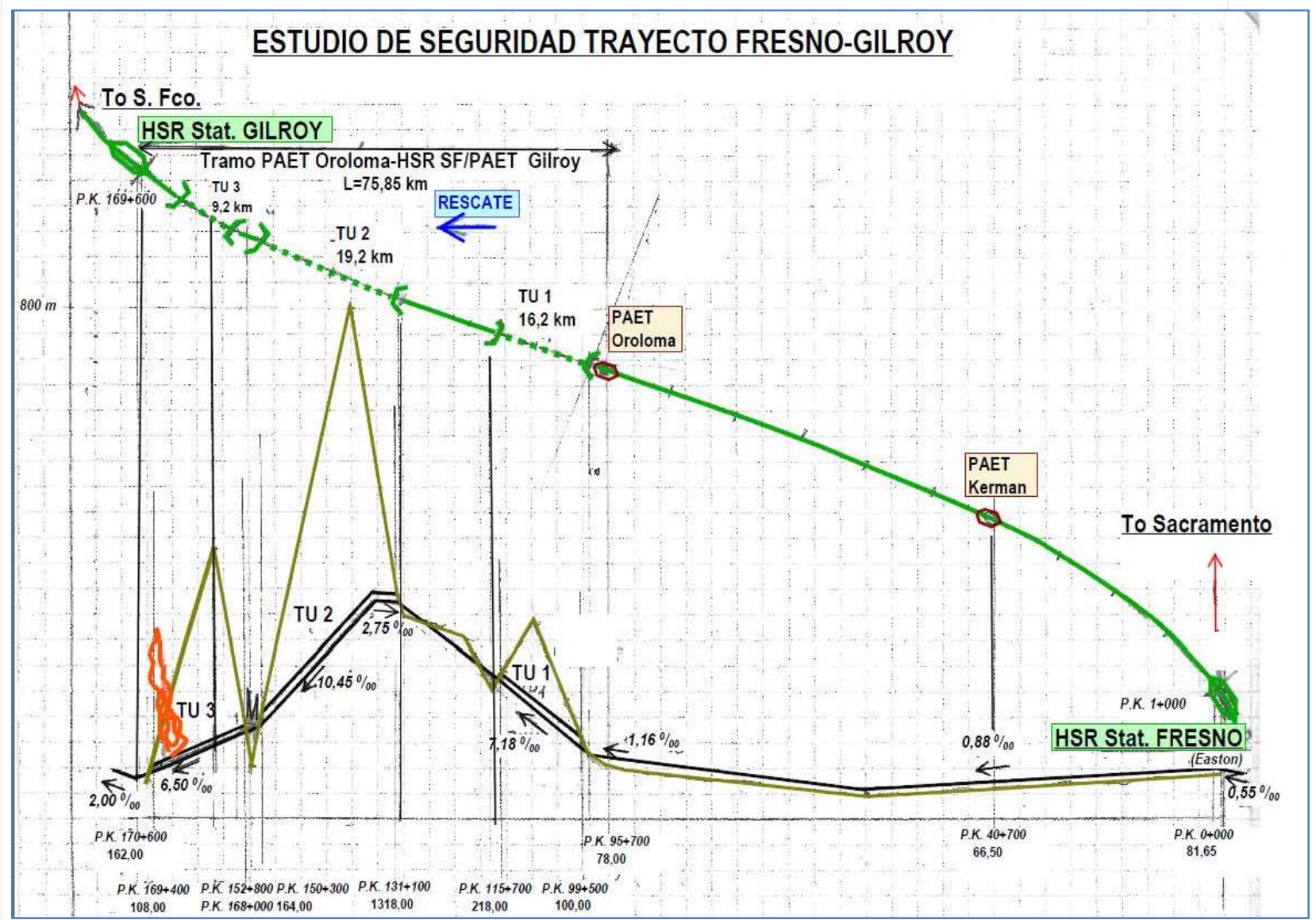

10800 P.K. $168+000164,00 \quad 1318,00 \quad 218,00 \quad 100,00$

Figura 7a: Plataforma VAL necesaria LAV/HSL Fresno- S.Fco. Airport (Farwest Project)

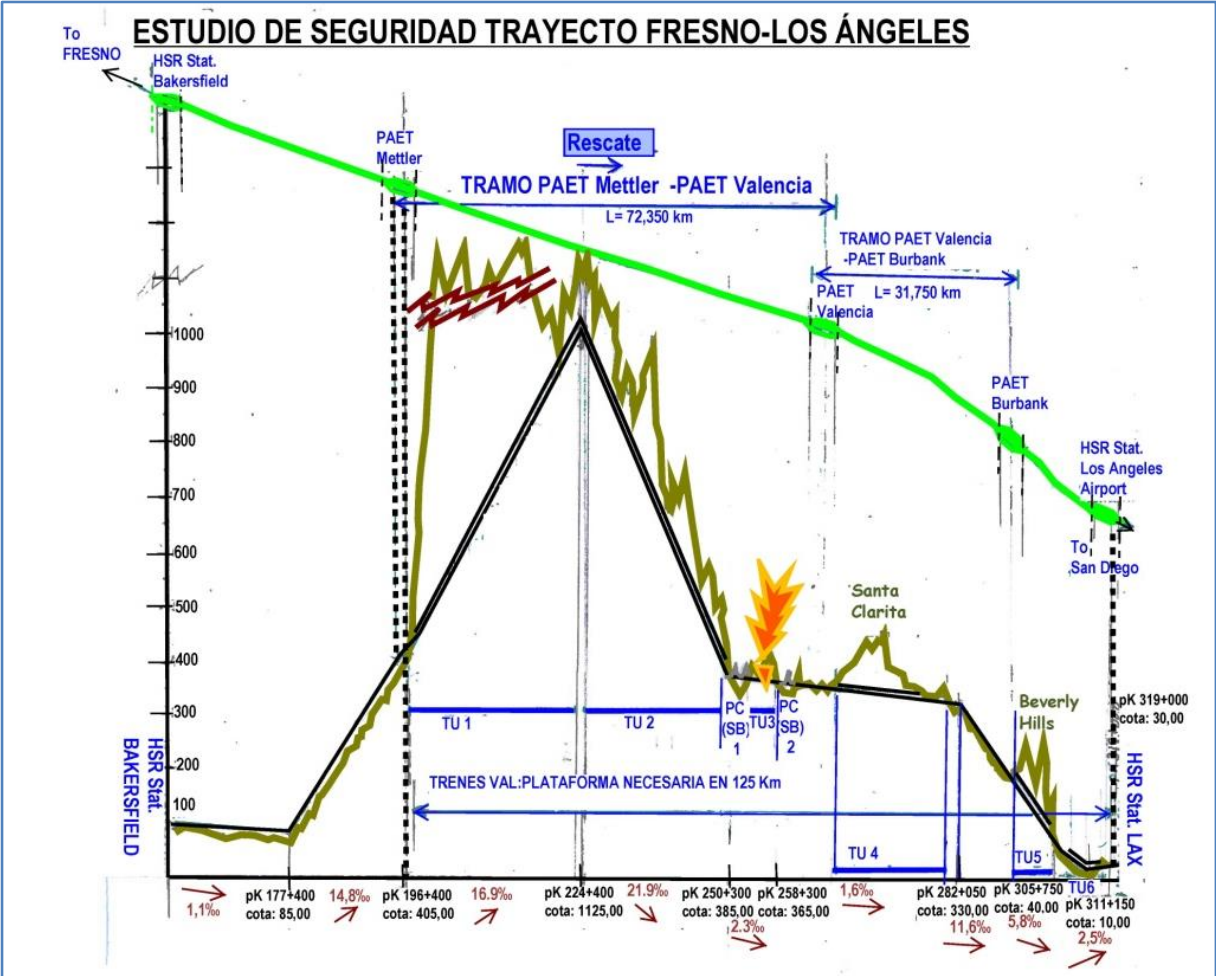

Figura 7b: Plataforma VAL necesaria LAV/HSL Fresno- Los Ángeles (Farwest Project) 


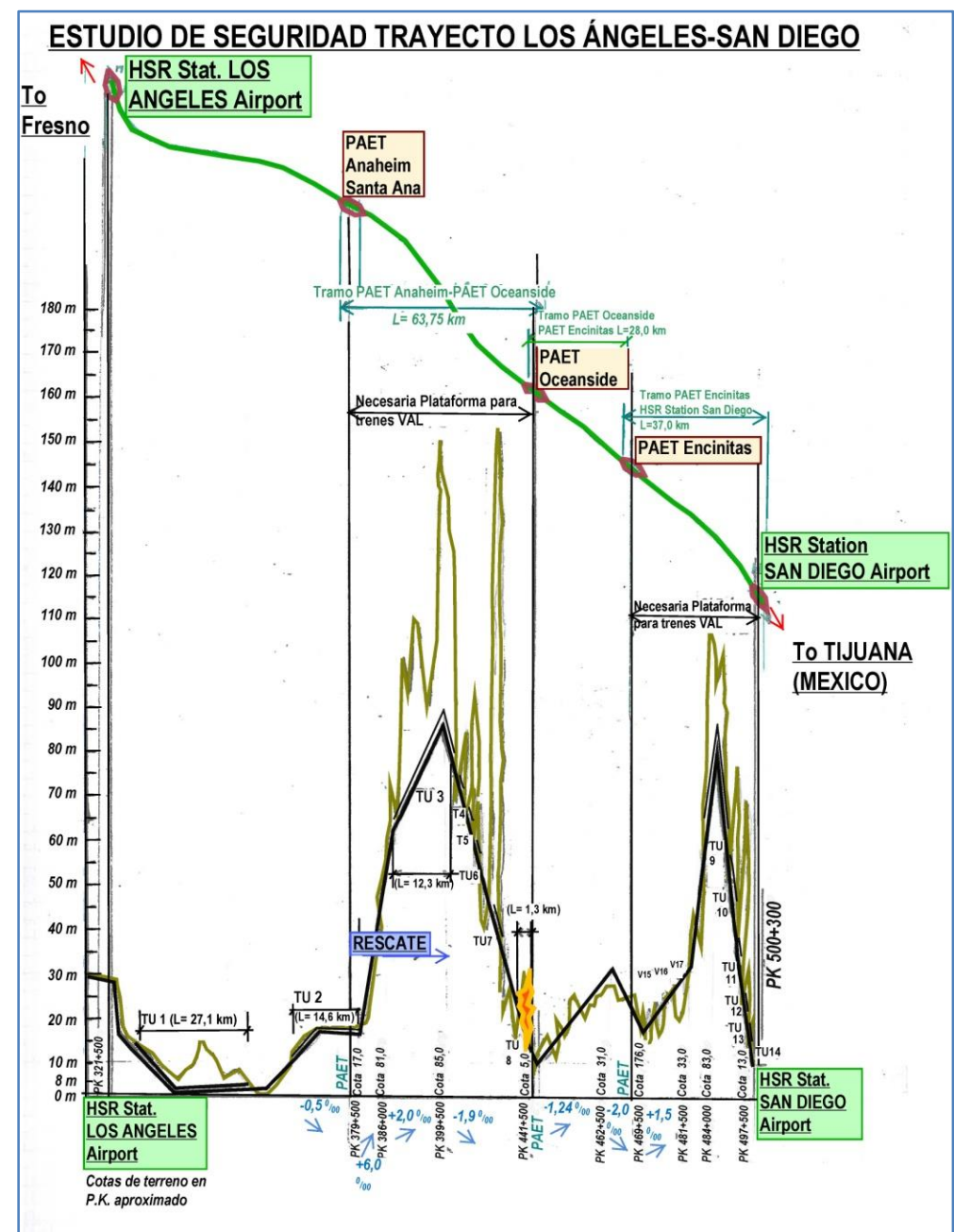

Figura 7c: Plataforma VAL necesaria LAV/HSL Los Ángeles-San Diego (Farwest Project)

Se comprueba, como era previsible, que el sobrecosto que lleva consigo esta medida de seguridad en el diseño de líneas de alta velocidad con grandes túneles en su trazado, depende principalmente del tamaño medio de los grandes túneles de la red estudiada, siendo directamente proporcional al mismo.(Coeficiente $A \alpha)$. Por otra parte, resultan también significativas las relaciones de Longitud de plataforma VAL "LVAL" a Longitud Total "L" (Coeficiente B $\beta$ ) y la proporción de plataforma en túnel "LTU" a la total "L" (Coeficiente $\mathrm{Cr})$.

Para aproximar los valores de estos coeficientes se analizan todas estas magnitudes y la descomposición en capítulos del Presupuesto de tres trayectos representativos de la Unidad estructural de la USHSRS "NUEVA ESPAÑA", tomadas del Proyecto Farwest, en dos de sus líneas: "Golden Gate Alternative: Fresno-San Francisco Airport" (Trayecto I:- PAET Oroloma (pk 99+500) - PAET/HSR Station Gilroy (pk170+600)) y "Missions Trail": a> "Tehachapi Section": Fresno-Los Angeles Airport" (Trayecto II:-PAET Mettler (pk 195+000) - PAET/HSR Station Los Angeles Airport (pk 320+000) y “Missions Trail”: b> Capistrano Section": Los Angeles Airport-San Diego Airport” (Trayecto III: 1>-PAET HSR Station (pk 378+250)-PAET Oceanside (pk 442+000) y 2>- PAET Encinitas-HSR Station San Diego).

En el trayecto I hay tres grandes túneles (TU1: 16,2 km; TU2: 19,2 km; TU3: 9,2 km)

En el Trayecto II también hay tres grandes túneles (TU1: 27,5 km; TU2: 25,6 km; TU3: 6,5 
$\mathrm{km})$

En el trayecto III se localiza solamente un gran túnel (TU9: 7,5 km)

El cuadro I adjunto "Anteproyectos de Prediseño de la USHSRS en NUEVA ESPAÑA (en rojo las incidencias con sobrecosto por plataforma VAL)" recoge en su primera columna las características, antes mencionadas, así como el resto de figuras representativas del Proyecto Farwest, necesarias para la estimación aproximada de sobrecosto por la aplicación de esta medida de diseño sostenible de mejora de la seguridad en trayectos con necesidad de grandes túneles en el resto de los anteproyectos en los que los autores han prediseñado las $31 \mathrm{LAV}$ de la unidad estructural USHSRS en NUEVA ESPAÑA (Figuras 8 y 9).

El planteamiento de la expresión: $\Delta=(\mathrm{A} \alpha) \mathrm{x}+(\mathrm{B} \beta) \mathrm{y}+(\mathrm{C} \gamma) \mathrm{z}$

en los tres trayectos tomados como referencia, por su representatividad, naturaleza geológica de su trazado y mayor fiabilidad en sus valoraciones, por haberse estudiado con detalle el trazado de los mismos, la definición de los grandes viaductos en ellos comprendidos y las estrategias de salvamento y rescate en los estudios específicos de seguridad, lleva a la estimación de los coeficientes propuestos como más representativos del sobrecosto de esta propuesta de diseño los que se reflejan en el cuadro adjunto, anteriormente citado.

El sobrecosto medio es del 5,4\% (11199/(218510-11199), que a nuestro juicio, puede considerarse rentablemente asumible para una explotación de la red con una mayor seguridad para los viajeros.

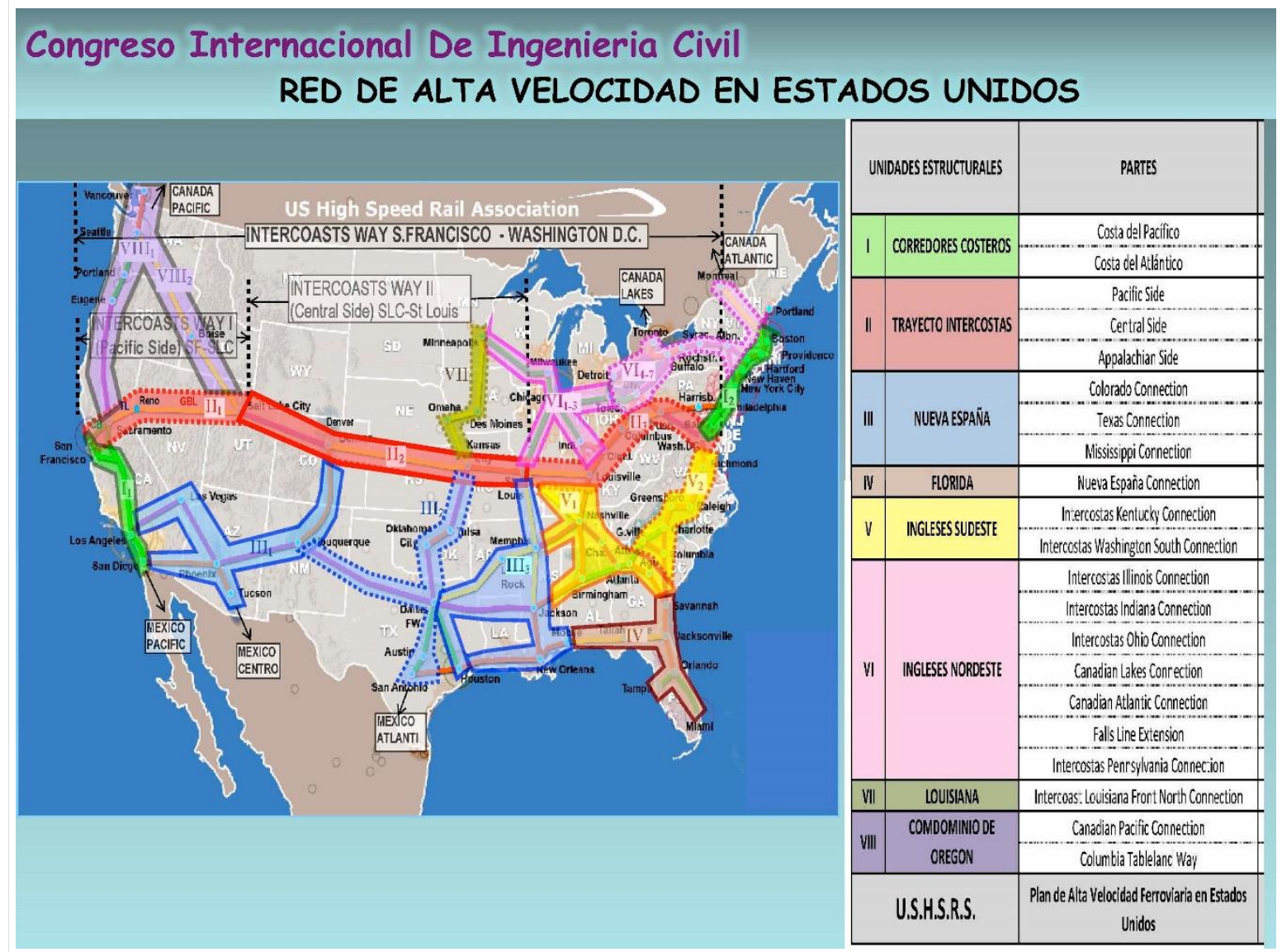

Figura 8. Unidades Estructurales consideradas en el desarrollo de la USHSRS 


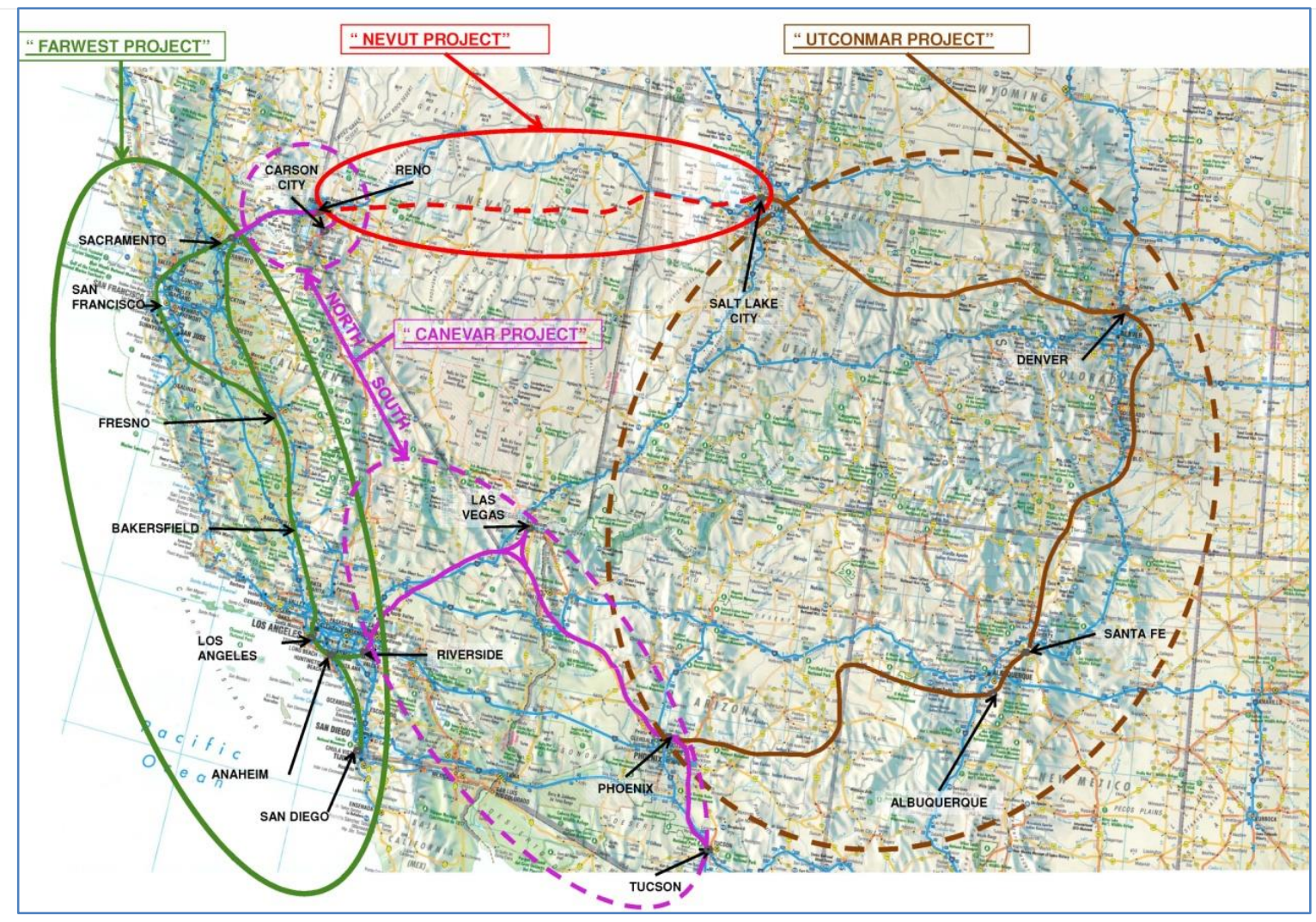

Figura 9a. Anteproyectos de Rediseño de la USHSRS en Nueva España I y II

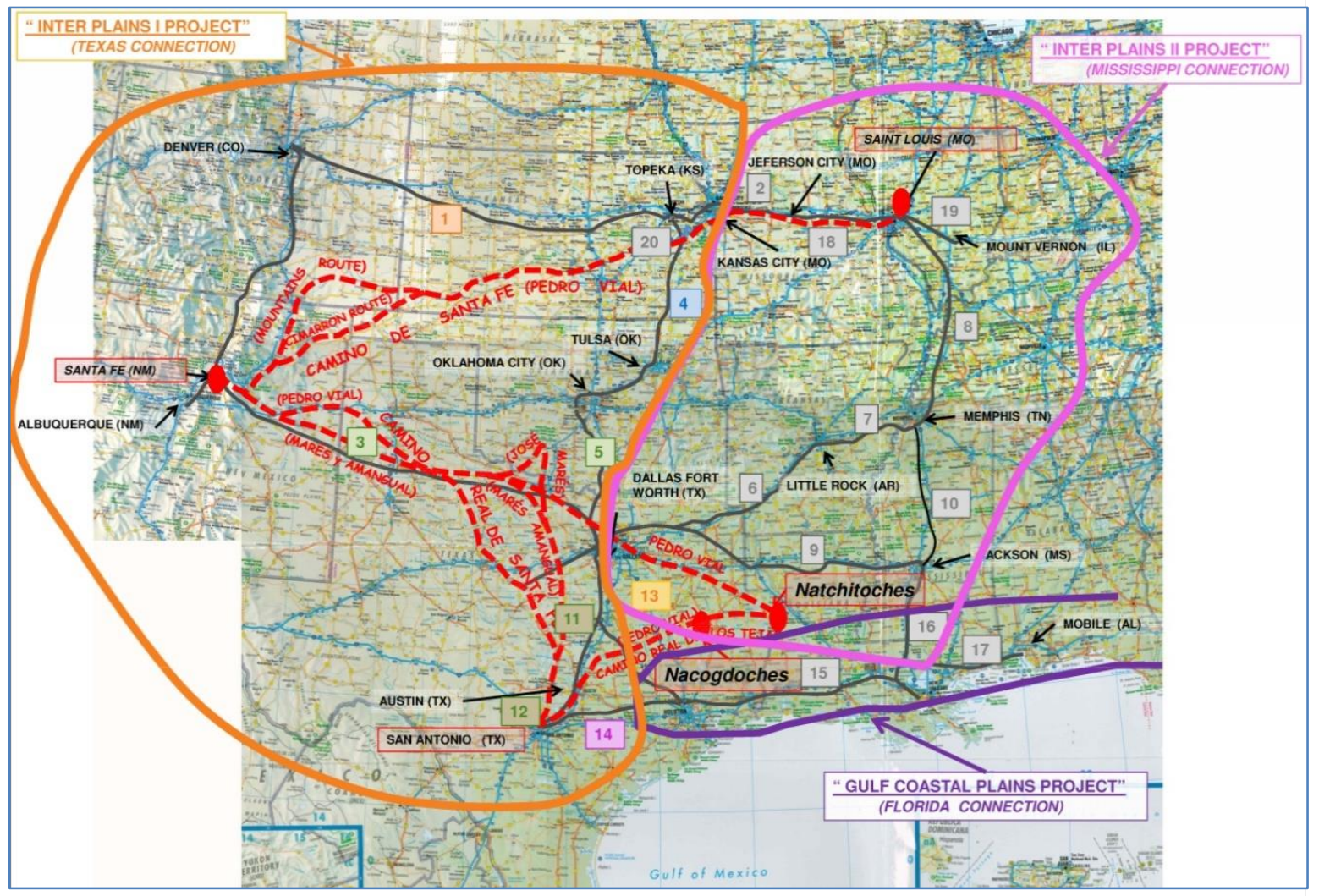

Figura 9b. Anteproyectos de Rediseño de la USHSRS en Nueva España III 


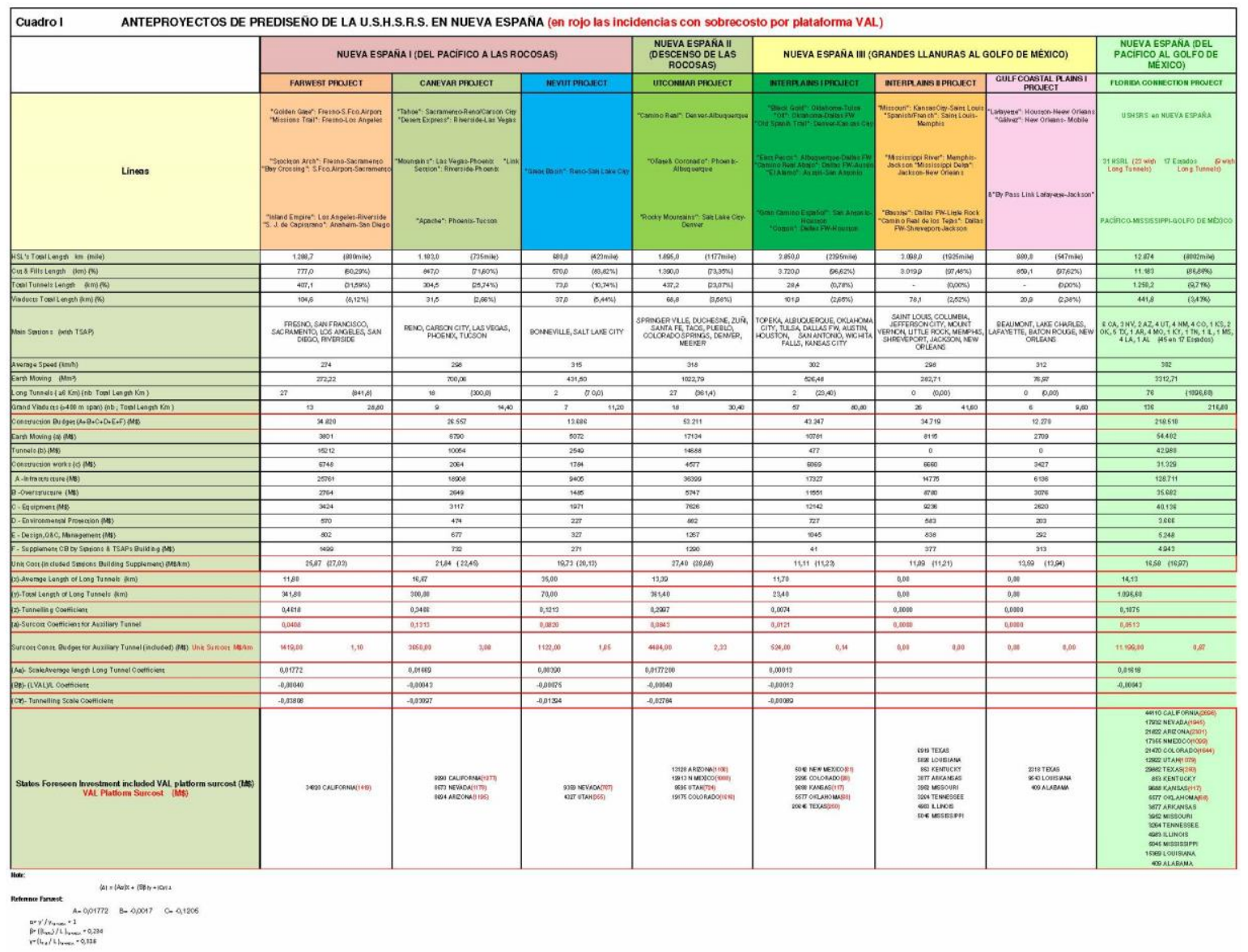

Esta consideración puede verse reforzada si extrapolamos el estudio hecho en el Proyecto Farwest, "Planteamiento sostenible de la Red de Alta Velocidad Ferroviaria en California (CHSRS) (Ref 3).

En dicho estudio se llegaba a que para la red del Estado de California $(1.288 \mathrm{~km})$, con la solución alternativa propuesta en el Proyecto Farwest, con un coste de construcción de $34.820 \mathrm{M} \$$, que incluye el coste derivado de la solución, como se ha explicado anteriormente, de disponer de grandes túneles, con sección grande para doble vía de trenes TAV y plataforma para trenes VAL, con túneles auxiliares, con una ocupación media de 12,36 Millones de viajeros/año, aplicando una tarifa media de $0,265 \$ / \mathrm{km}$, se alcanzaba un TIR del 6\% en Proyección Financiera de 50 años.

Un primer análisis de explotación de la red USHSRS en Nueva España, con 12.874 km y un coste de construcción de $218.510 \mathrm{M} \$$, que potencia un intercambio de un mundo de negocios de 40 Millones de usuarios potenciales (Ref 9), podría tener también un planteamiento sostenible (competitiva con otros medios de transporte), con una tarifa unitaria algo menor, del orden de $0,165 \$ / \mathrm{km}$, teniendo en cuenta que la longitud media de las líneas del Proyecto Farwest es de $214 \mathrm{~km}$ y las de Nueva España es de $415 \mathrm{~km}$. 


\section{REFERENCIAS}

1-Fort, L. (2004) "Safety. The essential environmental requirement.Applications to the design of railway infrastructures". Safety in High Speed railway tunnels (Spanish, English \& French) ISBN 8489456232 Cersa $2^{\text {a }}$ Ed. Presented in Prague. Madrid, Spain.

2-Fort, L. (2011) "Anteproyecto de Estructuras CHSRS Golden Gate Alternative" Colegio de Ingenieros de Caminos, Canales y Puertos Ref 146304. Madrid, Spain.

3-Díaz del Río, M, Fort, L. and Fort, C. (2011-2014) "First Part: Farwest Project";"Second Part: Golden Gate Alternative: HSL Fresno-San Francisco Airport";"'Third Part: Missions Trail Alternative: HSL Fresno- Los Angeles-San Diego/Riverside";"'Fourth Part: Stockton Arch Alternative: HSL Fresno-Sacramento";"Fifth Part: "Bay Crossing Alternative: HSL San Francisco Airport-Sacramento"; "Sixth Part: Sustainable Plan of the System (autofinancing operation:Fares)" Revista de Ingeniería Civil n ${ }^{\circ} \mathrm{s}$ 167; 169;170;172;173 CEDEX. Ministerio de Fomento. Madrid, Spain.

4-Fort, L. and Fort, C. (2014-2016) "Farwest", "Canevar", "Nevut", "Utconmar", "InterplainsI" "Interplains II" "Gulf Coastal Plains I" Projects Colegio de Ingenieros de Caminos, Canales y Puertos Refs: 148397, 149458, 150466, 150711, 151264, 151365. Madrid, Spain.

5-Fort, L. and Fort, C. (2015) "Viejos Caminos que inspiran los nuevos. La conexión de las redesHSR de los Estados de California, Nevada Y Arizona". Revista Fomento. Ministerio de Fomento ${ }^{\circ} 649$ Abril 2015. Madrid, Spain.

6-Fort, L. and Fort, C. (2016) "Del Pacífico a las Rocosas. Los Proyectos de desarrollo de las líneas de Alta Velocidad en Estados Unidos (I)" Revista Fomento. Ministerio de Fomento nº58 Febrero 2016. Madrid, Spain.

7-Fort, L. and Fort, C. (2016) “ Red de Alta Velocidad en Estados Unidos” I Congreso Internacional de Ingeniería Civil” Comunicación al Grupo I:Planes de Inversión y Mantenimiento de Infraestructuras. Colegio de Ingenieros de Caminos, Canales y Puertos, 2y3 de Marzo de2016. Madrid, Spain.

8-Fort, 1. and Fort, C. (2016) "El Descenso de las Rocosas. Los Proyectos de desarrollo de Líneas de Alta Velocidad en Estados Unidos (II)" Revista Fomento. Ministerio de Fomento (En impresión). Madrid, Spain.

9-Fort, L. and Fort, C. (2016) "Por las Grandes Llanuras al Golfo de Méxicoi". Los Proyectos de desarrollo de líneas de Alta Velocidad en Estados Unidos (III) Revista Fomento. Ministerio de Fomento (En impresión), Madrid, Spain. 